\title{
Analysis of variance methods for the design and analysis of Monte Carlo statistical studies
}

\author{
EDWARD L. WIKE and JAMES D. CHURCH \\ The University of Kansas, Lawrence, Kansas 66045
}

\begin{abstract}
It was proposed that the data from Monte Carlo statistical investigations be subjected to analysis of variance methods rather than the conventional techniques of tabling, graphing, and inspecting the data. Two examples in which analysis of variance methods were applied to published Monte Carlo studies were presented. It was suggested that balanced factorial designs should be used whenever possible in Monte Carlo studies so that analysis of variance methods would be directly applicable. Finally, three advantages of analysis of variance methods over conventional techniques were described and analysis of variance methods were contrasted with a possible alternative analytical method, multiple regression analysis.
\end{abstract}

Monte Carlo studies can be valuable for assessing the null hypothesis, power, and robustness characteristics of statistical tests. Early Monte Carlo investigations (e.g., Box \& Andersen, 1955; Norton, Note 1) which used devices like sorters and tabulators were laborious undertakings. With the advent of powerful high-speed computers, the potentiality for Monte Carlo studies increased dramatically. One important consequence of this enhanced potentiality is that investigators doing Monte Carlo computer experiments may become inundated by data, with the attendant problem of understanding their meaning. This problem was confronted in a Monte Carlo study on the properties of homogeneity of variance tests (Church \& Wike, 1976), and other investigators (e.g., Hardyck, Note 2) have reported similar experiences. Usually, the data from Monte Carlo studies have been tabled, graphed, and inspected in efforts to extract their meaning. These methods are probably satisfactory for the discovery of gross trends in such data. However, they are relatively crude and subjective methods and they may fail to detect more subtle and complex relationships.

The basic data in our variance study were the proportions of null hypothesis rejections at the .05 and .01 level of significance per 500 or 1,000 computer runs. We applied the conventional analytical methods of tabling, graphing, and inspecting these rejection proportions. Then it became apparent that in half of the investigation we had inadvertently varied orthogonally the independent factors of numbers of samples, sample sizes, tests, and distributions. This discovery provided a meaningful shorthand description to others as to what was done in this half of the Monte Carlo study. Later, we asked: If we have a factorial design, why not submit the rejection proportions to an analysis of variance? In other words, why not analyze the data from the Monte Carlo study as a factorially designed experiment in order to evaluate the significance of the factors that were manipulated in the investigation? The soundness of this approach rests upon the well-established robustness of fixed-effects analysis of variance techniques (Glass, Peckham, \& Sanders, 1972). Still later, we asked: Why not determine the magnitudes of effects by determining percentages of accounted-for variance? To demonstrate the utility of these analysis of variance approaches to Monte Carlo data, two examples are considered below using some of our data and some from Miller (1968).

\section{EXAMPLE 1}

\section{Method}

In the portion of the variance study mentioned above, $\mathrm{k}$ random samples ( 3 and 6 ) of size $n$ ( 5 and 15) were drawn from 11 distributions (quasi-normal, gammas, and gamma mixtures) having equal variances. Five homogeneity of variance tests (Hartley's $\mathrm{F}$ maximum test, Bartlett's test, the $\mathrm{k}$-sample BoxAndersen test, Levene's $z$ test, and the jackknife test) were applied to the random sample values and the resulting proportions of rejections of the null hypothesis at $\alpha=.05$ per 1,000 computer experiments were obtained. Thus, a 2 by 2 by 11 by 5 data matrix with one observation (rejection proportion) per cell resulted, and these data were subjected to a fixed-effects analysis of variance in which the highest order interaction served as the error term. ${ }^{1}$

\section{Results}

The analysis of variance revealed that 12 out of the 14 possible effects were significant at less than the .01 level. We attributed the large number of significant differences to the potency of the independent variables and to the fact that each rejection proportion included in the analysis was highly reliable as a consequence of its being based upon 1,000 computer runs. To gain additional information regarding the relative magnitudes of the significant effects, the percentages of accounted-for variance were calculated by Hays' formulas (1963, p. 407). This analysis showed that three effects, distributions, tests, and their interaction, accounted for $51 \%$, $12 \%$, and $18 \%$ of the total variance, respectively, and that no other effect accounted for more than $3 \% .^{2}$ In 
view of these results it would seem appropriate to summarize and display the data with a Tests by Distributions interaction table (see Church \& Wike, 1976, Table 2). More analytical conclusions can then be drawn from breakdowns of this interaction table by simple main effects tests (i.e., comparing variance tests within each distribution or distributions within each variance test or both) and by follow-up multiple comparisons tests in the instances of significant simple main effects. To illustrate these procedures, consider the variance tests within the exponential distribution (a gamma distribution with the shape parameter $r=1$ and the scale parameter $\lambda=1$ ). At $\alpha=.05$, the expected null hypothesis rejection proportions for the variance tests should be .050 . The obtained values averaged across $n$ and $k$ for the jackknife, BoxAndersen, Levene, F maximum, and Bartlett tests were $.110, .120, .253, .345$, and .397 , respectively. Thus, the jackknife test performed the best with this exponential distribution and Bartlett's test the worst. Since the simple main effects test was highly significant $(\mathrm{p}<.001)$, pairwise comparisons of the average rejection proportions for the five variance tests were made with Tukey $b$ tests (Winer, 1971). The Tukey b pairwise tests showed that all comparisons among the tests were significant $(p<.01)$ except for the comparison between the jackknife and Box-Andersen tests, which did not differ from one another.

\section{EXAMPLE 2}

\section{Method}

Miller (1968) did two Monte Carlo studies to evaluate the performance of a number of two-sample homogeneity of variance tests. In the first study the standard $F$ test and six other purportedly robust tests were investigated using five distributions (uniform, normal, double exponential, skew double exponential, and sixth power distribution), five variance ratios $(1: 1$, $2: 1,4: 1,6: 1$, and $10: 1), \alpha=.05$ and .01 , and sample sizes of 25 . The resulting null hypothesis rejection proportions per 1,000 computer runs were tabled and conclusions were drawn by "inspection of the table." As a consequence of this inspection, three of the tests were discarded in a second study with sample sizes of 10 .

To contrast the inspection technique with our approach, a 2 by 5 by 4 by 4 analysis of variance was done with one rejection proportion per cell for $\alpha=.05$ with the data from the two Miller studies combined. The independent variables were: sample sizes (10 and 25); distributions (as described above); tests ( $F$ test, Box-Andersen test, jackknife test, and Levene's s test); and variance ratios (the four unequal variance conditions described above). In this analysis, we are concerned with the power performance of the tests.

Since previous research and Miller's own data have shown conclusively that the $F$ test for homogeneity of variance is markedly nonrobust, a second analysis of variance was then performed with the $F$ test omitted.

\section{Results}

The first analysis of variance disclosed that all effects were significant at less than the .01 level except for the interactions of Tests by Sample Sizes by Distributions and Tests by Distributions by Variance Ratios. Again, the percentages of variance accounted for by the significant factors were calculated, and it was found that $95 \%$ of the total variance could be attributed to five effects: variance ratios (53\%), distributions (19\%), sample sizes $(14 \%)$, tests $(5 \%)$, and tests by distributions (4\%). The second analysis without the $\mathrm{F}$ test revealed a similar pattern of significant results but now four effects accounted for $96 \%$ of the variance. These effects were: variance ratios (49\%), distributions (29\%), sample sizes (15\%), and variance ratios by distributions by sample sizes (3\%). Thus, with the F test excluded, tests and the interactions of tests with the other factors proved to be trivial sources of accounted-for variance. The largest of these effects was tests, which "explained" only $1 \%$ of the total variance.

What conclusions regarding power did Miller draw by inspection of his data and how do these conclusions compare with the results from the analysis of variance techniques? Miller (1968, p. 579) concluded that the results of his small sample study (i.e., $n=10$ ) were very similar to those from his first study $(\mathrm{n}=25)$. In particular, he concluded that the $F$ test was nonrobust, the Box-Andersen and jackknife tests were about equal in power (the former test being slightly better at the .05 level and the reverse being true at the .01 level), and that, while the Levene $s$ test was robust, it was far less powerful than the Box-Andersen and jackknife tests. Although we do not question the validity of these conclusions, the analysis of variance approach suggests that when the $F$ test was excluded the magnitude of the effects of tests upon power was quite small in comparison to the influence of the other factors in Miller's design, such as the variance ratios, distributions, and sample sizes. Obviously it is trivial to conclude that larger variance ratios and larger sample sizes result in larger proportions of null hypothesis rejections. However, the analysis of variance methods also disclosed that the types of distributions had a far more profound effect upon power than did tests. Such a conclusion is not readily ascertainable from inspection of the data. As suggested in Example 1, the interested investigator could do additional comparisons to explore the differences among the distributions or the three-way interaction involving distributions.

\section{DISCUSSION}

The purpose of this note was to suggest new techniques for analyzing the results of Monte Carlo statistical studies. In the two examples which were presented, the techniques appeared promising. In Example 1, the application of the techniques led to the reduction of the results from a complex Monte Carlo study on k-sample tests for homogeneity of variance to a single two-way interaction table. In Example 2, it was demonstrated that when the $\mathrm{F}$ test was omitted from the design the type of two-sample homogeneity of variance test was much less important than other factors that were included in the design. Although the two examples involved Monte Carlo studies of tests of homogeneity of variance, the suggested analytical techniques are, of course, appli- 
cable to Monte Carlo studies of other statistics as well. The only restriction to the use of the techniques is that the Monte Carlo study be an orthogonal design. Accordingly, there is an implication here also for the design of Monte Carlo statistical investigations: Whenever possible the investigator should arrange his independent factors so as to form a fully balanced design. Then the techniques proposed here are directly applicable. However, even the data from unbalanced designs can sometimes be subjected to a series of analysis of variance tests in which balanced segments are selected from a larger nonorthogonal design.

In the two examples the analysis of variance techniques seemed useful because the total variance in both studies was found to be accounted for in terms of a few factors and interactions. In other examples that we have analyzed, the results were more complicated, with more factors accounting for smaller percentages of the total variance. In these cases, however, one is not dealing with a deficiency in the analytical techniques but simply with a more complex empirical situation.

Should the inspection of Monte Carlo data be abandoned in favor of analysis of variance techniques? Certainly not! The Monte Carlo investigator needs to constantly check his output data to decide whether or not he is obtaining reasonable data, what levels of the independent variables to include in his design, when to stop doing computer runs, etc. Similarly, inspection of tabled results can be valuable, as well as the comparison of such results with those from other investigations. On the other hand, the analysis of variance methods appear to offer a number of advantages over the simple inspection-of-tables technique. (1) They provide a way of "looking at" all of the factors and combinations of factors in a design simultaneously in an objective and repeatable fashion. (2) Hunches as to differences in the data obtained by inspection are replaced by statistical significance tests of differences. (3) Indications regarding the relative magnitude of the effects in Monte Carlo studies can be attained.

Finally, mention should be made of an alternative set of statistical techniques for analyzing the data from Monte Carlo statistical investigations. As Cohen and Cohen (1975) have indicated, analysis of variance is a special case of multiple regression analysis. It follows that the significance of main effects and interactions as well as the percentages of accounted-for variance can be obtained also by multiple regression analysis. One disadvantage of the multiple regression approach, however, is that complex factorial designs require a large number of independent variables to represent the design. For instance, in Example 1, it would require four independent variables, $\left(\mathrm{X}_{1} \ldots \mathrm{X}_{4}\right)$ to represent the five variance tests, 10 independent variables $\left(\mathrm{X}_{5} \ldots \mathrm{X}_{14}\right)$ to represent the 11 distributions, and 40 independent variables $\left(X_{1} X_{5} \ldots X_{4} X_{14}\right)$ to represent the interaction of Tests by Distributions. Thus, for complex designs the multiple regression method becomes a less efficient algorithm than analysis of variance. In fact, some complex factorial designs may exceed the limits of existing multiple regression programs. Cohen and Cohen have argued that investigators should limit their analyses to main effects and lower order interactions (three-way at the most) in order to reduce the number of independent variables in multiple regression analysis of complex factorial designs. At the very least, it would appear that multiple regression analysis is a suitable alternative method for the analysis of Monte Carlo data when the investigator has a simple design and is interested in main effects and a few lower order interactions only.

\section{REFERENCE NOTES}

1. Norton, D. W. An empirical investigation of some effects of non-normality and heterogeneity on the $F$ distribution. Unpublished doctoral dissertation, University of Iowa, 1952.

2. Hardyck, C. D. Personal communication. May 10, 1976.

3. Chambers, D. S. Personal communication. May 9, 1976.

\section{REFERENCES}

Box. G. E. P., \& Andersen, S. L. Permutation theory in the derivation of robust criteria and the study of departures from assumption. Journal of the Royal Statistical Society, Series B, 1955, 17, 1-26.

Butler, D. H., Kamlet, A. S., \& Monty, R. A. A multi-purpose analysis of variance FORTRAN IV computer program. Psychonomic Monograph Supplements, 1969, 2, No. 16(Whole No. 32).

ChURCH, J. D., \& WiKe, E. L. The robustness of homogeneity of variance tests for asymmetric distributions: A Monte Carlo study. Bulletin of the Psychonomic Society, 1976, 7, 417-420.

COHEN, J., \& Cohen, P. Applied multiple regression/correlation analysis for the behavioral sciences. Hillsdale, N.J: Lawrence Erlbaum, 1975.

Glass. G. V., Peckham, P. D., \& Sanders, J. R. Consequences of failure to meet assumptions underlying the fixed-effects analysis of variance and covariance. Review of Educational Research, 1972, 42, 237-288.

Ha Ys, W. L. Statistics for psychologists. New York: Holt, Rinehart and Winston, 1963.

Miller, R. G., JR. Jackknifing variances. Annals of Mathematical Statistics, 1968, 39, 567-582.

WINER, B. J. Statistical principles in experimental design (2nd ed.). New York: McGraw-Hill, 1971.

\section{NOTES}

1. We found that the ANOVAR program (Butler, Kamlet, \& Monty, 1969) performed these analyses optimally. The core requirements for the SPSS analysis of variance program were excessive and the BMD P2V program was about 50 times more costly than ANOVAR. Neither ANOVAR nor P2V will output IF ratios when there is only one observation per cell.

2. At the suggestion of Professor David S. Chambers (Note 3), these data were subjected to a common logarithm transformation and reanalyzed. Since the percentages of accounted-for variance for the same three effects were $53 \%, 15 \%$, and $15 \%$, respectively, it was concluded that the results were largely invariant under this transformation.

(Received for publication March 31, 1977.) 